\title{
A criação de GIFs com o GeoGebra para produção de narrativas matemáticas digitais
}

The creation of gifs with the Geogebra for the production of digital mathematical narratives

Carla Vital ${ }^{1}$

Ricardo Scucuglia Rodrigues da Silva²

\section{Resumo}

Neste artigo, discutimos aspectos sobre processo de criação de GIFs com o software GeoGebra. Esses GIFs foram criados por grupos de professores e futuros professores de Matemática em um curso de extensão universitária sobre Geometria. Os GIFs foram utilizados na produção de uma Performance Matemática Digital (PMD). Portanto, esta pesquisa explora uma interlocução entre o uso de tecnologias digitais e artes em Educação Matemática. Este estudo é de natureza qualitativa e a produção de dados ocorreu por meio de registros audiovisuais do referido curso, considerando-se o ambiente de interação e as telas dos computadores utilizados pelos participantes. Os resultados evidenciam o papel das tecnologias e das artes na produção de significados matemáticos. Nossa análise conclui que a produção de conhecimentos e aprendizagem matemática, bem como o engajamento dos participantes, foram aprimorados pelas potencialidades tecnológicas do GeoGebra e por elementos estéticos das construções dinâmicas.

Palavras chave: GeoGebra; Experimentação; Seres-humanos-com-mídias.

\section{Abstract}

In this article, we discuss the process of creating GIFs with the software GeoGebra. These GIFs were created by groups of mathematics teachers and pre-service teachers in a knowledge mobilization course on Geometry. GIFs were used in the production of a Digital Mathematical Performance (DMP). Therefore, this research explores the intersection of the use of digital technologies and the arts in Mathematics Education. This study has a qualitative design, and the data were produced through audio-visual recordings of the sessions of the course, taking into account the learning environment and the screens of the computers used by the participants. The results emphasize the role of technologies and the arts in the production of mathematical meanings. Our analysis concludes that the productions of mathematical knowledge and learning, as well as participants' engagement, were enhanced by the technological potentialities of Geogebra and the aesthetic elements of the dynamic constructions.

Keywords: GeoGebra; Experimentation; Humans-with-media.

\footnotetext{
${ }^{1}$ Universidade Estadual Paulista | professoracarlavital@gmail.com

${ }^{2}$ Universidade Estadual Paulista | ricardo.scucuglia@unesp.br
} 


\title{
Introdução
}

Neste artigo apresentamos resultados de uma pesquisa que investigou o processo de produção de Performances Matemáticas Digitais (PMDs) envolvendo o uso do software GeoGebra. Em específico, exploramos a criação de GIFs produzidos com o GeoGebra. Neste cenário, PMDs podem ser concebidas como narrativas matemáticas digitais nas quais artes (performáticas) são utilizadas para comunicação e representação matemática em cenários educacionais. Trata-se de uma interlocução entre o uso de tecnologias digitais, em particular a produção audiovisual, e o uso pedagógico de expressões e performances artísticas como o cinema, a música, a dança e a poesia em Educação Matemática.

De acordo com Borba, Scucuglia e Gadanidis (2014), estamos vivenciando atualmente uma quarta fase no que se refere ao uso de tecnologias digitais em Educação Matemática. Essa fase é caracterizada pelo uso do software GeoGebra, de tecnologias portáteis (notebooks, smartphones, câmeras de vídeos, tablets) e de Internet de alta velocidade (FARIA; ROMANELLO; DOMINGUES, 2018). A atual fase do uso de tecnologias também envolve questões relacionadas às Artes. Nesse sentido, a noção denominada PMD tem sido explorada no contexto de pesquisas que investigam o uso integrado de Artes e tecnologias digitais em Educação Matemática em diversificados níveis de ensino (SCUCUGLIA; GADANIDIS, 2013).

As pesquisas sobre PMDs tiveram início em 2006 visando integrar essas temáticas e levá-las para a sala de aula a fim de (des)construir imagens sobre a Matemática, usualmente vista como fria, exata e formalista (GREGORUTTI, 2016). Além disso, pretendia-se que os matemáticos e educadores matemáticos pudessem atribuir um significado diferenciado para a expressão performance na área de Educação ou Ensino, a qual está associada ao desempenho ou rendimento em testes. Nesse sentido, buscou-se uma lente artística para se conceber processos de comunicação, representação, ensino e aprendizagem matemática (GADANIDIS; BORBA, 2006).

\begin{abstract}
Performance Matemática Digital pode ser entendida como um objeto digital, o qual é utilizado para comunicar ideias e estéticas. Expressar ideias matemáticas em forma de narrativa multimodal é consistente com a noção de não domesticação de novas mídias, na medida em que é uma forma de expressão que dificilmente poderia ser realizada sem a presença do ator Internet em um coletivo de seres-humanos-com-mídias (BORBA; SCUCUGLIA; GADANIDIS, 2014, p. 128).
\end{abstract}

Nesse contexto, estudos têm sido desenvolvidos sobre essa temática, principalmente no Canadá (SCUCUGLIA, 2012). No Brasil, PMD é uma tendência ainda em fase de consolidação como linha de pesquisa e/ou metodologia de ensino em Educação Matemática (BORBA; SCUCUGLIA; GADANIDIS, 2014). Alguns estudos em nível de mestrado vêm sendo desenvolvidos (LACERDA, 2015; VITAL, 2018), dentre outros níveis (SCUCUGLIA, GREGORUTTI, 2015). A maioria das PMDs produzidas nessas pesquisas são vídeos digitais nos quais estudantes e (futuros) professores utilizam a música, a dança, o teatro, o cinema e/ou a poesia para comunicarem suas ideias matemáticas. Na pesquisa relatada neste artigo, buscamos investigar aspectos acerca do uso do software GeoGebra na produção de PMDs e, em específico, o uso do recurso Graphics Interchange Format (GIF). Os participantes deste estudo foram professores e futuros professores de Matemática, os quais participaram de um curso de extensão universitária sobre GeoGebra e PMDs. 
PMDs podem também ser concebidas como narrativas matemáticas artísticas-digitais. Doxiadis (2003) discute o papel das narrativas matemáticas, argumentando que, tradicionalmente, a Matemática "é o protótipo de uma ciência lógico-dedutivo [cuja] [...] principal tarefa é uma classificação completa de seus objetos de investigação" (DOXIADIS, 2003 , p. 7). Em contraste, "narrativas - como toda a arte - nos falam apenas na medida em que somos tocados por elas" (DOXIADIS, 2003, p. 8). Dessa maneira, a exploração de narrativas em Matemática é vista como uma "revolução".

Narrativas matemáticas devem entrar no currículo escolar, na educação
primária e secundária. O objetivo é: a) aumentar o apelo da disciplina, b)
oferecer um sentido de relevância intelectual, histórica e social e um lugar
na nossa cultura, c) oferecer aos alunos uma melhor noção do alcance do
campo da Matemática, além de técnicas [tradicionais]... Então,
professores, utilizem parte de seu tempo ensinando com narrativas, para
incorporar a Matemática à alma. (DOXIADIS, 2003, p. 20).

Práticas de investigação narrativa também assumem papéis pedagógicos importantes no ensino de Matemática (ARAGÃO; GONÇALVES, 2005). Em particular, narrativas digitais, como PMDs, demonstram potencial semiótico alternativo, visto que, usualmente, processos de ensino, aprendizagem e avaliação em sala de aula de Matemática são hegemonicamente pautados na linguagem escrita. Nesse sentido, fundamentados em Walsh (2010), temos explorado a noção de multimodalidade para evidenciar o fazer matemático-tecnológicoartístico emergente com a produção de PMDs em ambientes formativos (SCUCUGLIA, 2014, 2015).

Além disso, destacamos neste cenário a noção denominada seres-humanos-commídias (BORBA; VILLARREAL, 2005). Buscamos investigar o papel das tecnologias no condicionamento da produção de conhecimentos matemáticos, ou seja, o papel das mídias na produção de significados e na aprendizagem matemática. Por esse motivo, em nossas considerações analíticas discutiremos questões sobre experimentação-com-tecnologias e visualização, ou seja, processos inerentes ao pensar-com-tecnologias na constituição de coletivos pensantes de seres-humanos-com-GeoGebra. O GeoGebra, nesse sentido, assume um papel tecnológico e semítico protagonista na produção de conhecimentos matemáticos (URDANETA; GONZÁLEZ; CASTILLO, 2017). Em particular, exploraremos também o papel das artes (elementos estéticos) em relação a processos de pensamento matemático no referido cenário.

É pertinente ainda destacarmos o potencial pedagógico dos GIFs animados na aprendizagem matemática. GIF é um tipo de formato de arquivo digital criado aproximadamente no final da década de 1980 com o intuito de compartilhar rapidamente arquivos de imagem comprimidos. Com a evolução da Internet e da informática os GIFs passaram a compilar uma sequência de várias imagens que dão a ilusão de movimento, gerando assim os GIFs animados (NADAL, 2014). Apesar do GIF ser um tipo de arquivo atualmente muito utilizado pelos usuários da Internet, principalmente em redes sociais, uma de suas limitações técnicas é a baixa qualidade de resolução, cujo padrão de imagem é de apenas 8 bits (ALVES, 2015). No entanto, considerando o aspecto da contínua repetição que fomenta a percepção da noção de movimento de curta duração, o GIF possui um caráter visceral, considerado essencial em PMDs (BOORSTIN, 1990; SCUCUGLIA, 2012; VITAL, 2018).

Weiner (2010) argumenta que tal característica é eficiente para administrar o tempo de usuários na Internet já que o tempo utilizado para visualizar um GIF (segundos) é 
consideravelmente menor do que para ver um vídeo digital comum. Além disso, existem diversas outras características que os GIFs oferecem à audiência e que os diferenciam de outros produtos digitais ou mídias.

\begin{abstract}
A repetição das imagens no cotidiano expande seu contato com os indivíduos, desta maneira, o leitor consegue ultrapassar a apresentação inicial na qual a imagem parece ser delimitada por seus elementos plásticos. Nesse movimento, o indivíduo passa a encontrar diferentes camadas de sentido (...). No caso do ciberespaço, a multiplicidade de pontos de interação e a velocidade da transmissão da informação permitem trocas constantes entre os textos, revelando uma torrente progressiva de sentidos. No caso dos GIFs, a linguagem imagética é condicionada pelos aspectos técnicos do formato (há necessidade de um aparelho digital para sua leitura, computador/celular; a imagem é adaptada à resolução, ao tamanho e à codificação do GIF). (NADAL, 2014, p. 9).
\end{abstract}

A visualização fomentada pelos GIFs é de considerável importância pedagógica, pois a comunicação se estabelece por meio de elementos semióticos diversos (gráficos, espaços, movimentos, alfabetos, outros). De maneira geral, a visualização é uma característica pedagógica significativa do GeoGebra, possibilitando a interpretação de diferentes linguagens ou modos de comunicação que ele apresenta: gráficos, tabelas, expressões algébricas, formas geométricas, dentre outros. Além da exploração de elementos artísticos como simetrias, padrões, cores e formas (ZALESKI FILHO, 2013; MORAES, 2020), o GeoGebra possibilita também a exploração de elementos estéticos (SINCLAIR, 2004). Atividades criadas no GeoGebra que possuem o uso de controle deslizante podem ser salvas em arquivos formato. GIF, gerando a respectiva animação referente a dinamicidade do controle deslizante.

\title{
Metodologia
}

Esta pesquisa é de natureza qualitativa (BICUDO, 2005). De maneira geral, buscamos respostas a seguinte pergunta diretriz: como professores e futuros professores de Matemática produzem PMDs envolvendo o uso do software GeoGebra? Especificamente, considerando a noção denominada estudo de caso qualitativo (STAKE, 1999), exploramos neste artigo a seguinte questão: como os participantes da pesquisa construíram os GIFs utilizados na produção de uma PMD?

Os dados foram produzidos por meio da realização de um curso de extensão universitária de 18 horas oferecido em uma universidade pública do estado de São Paulo à professores e futuros professores de Matemática. O curso contou com a participação de 8 inscritos, 3 mediadores (alunos de pós-graduação) e 2 docentes responsáveis. $O$ título do curso foi "Introdução ao GeoGebra e Performance Matemática Digital" e todas as sessões, realizadas em um laboratório de informática, foram filmadas (tanto as interações no ambiente com câmera digital quanto as telas dos computadores por meio de uso do software livre aTube(atcher). O curso foi estruturado em 6 módulos, os quais propunham atividades sobre Geometria a serem exploradas com o GeoGebra. As atividades incluíam a construção de GIFs e a produção de PMDs. Os participantes se organizaram em 3 grupos, 
os quais nos referiremos neste artigo como Grupo A, Grupo B e Grupo C. Cada grupo criou 2 GIFs (GIF 1A, GIF 2A; GIF 1B, GIF 2B; GIF 1C, GIF 2C).

Os registros em vídeo foram analisados com base no modelo analítico proposto por Powell, Francisco e Maher (2004), o qual é constituído pelos seguintes procedimentos: observação, descrição, identificação de eventos críticos, transcrição, codificação, construção de enredo e composição da narrativa. Do ponto de vista ético, todos participantes assinaram carta de consentimento de participação em pesquisa. Nas transcrições dos dados, utilizamos os nomes reais dos participantes que concederam tal permissão e utilizamos nomes fictícios para os participantes que manifestaram essa preferência.

Cabe destacar que, em específico, consideramos os seguintes aspectos em relação à experimentação-com-tecnologias em nossa análise:

- Criação e simulação de modelos matemáticos;

- Geração de conjecturas matemáticas;

- Exploração de diversificadas formas de resolução;

- Manipulação dinâmica de objetos construídos;

- Realização de testes, conjecturas, utilizando um grande número de exemplos, modificando representações de objetos, simulando componentes de construções, etc.;

- Convencimento sobre a veracidade das conjecturas;

- Elaboração de novos tipos de problemas e construções matemáticas;

- Criação e conexão entre diferentes (e múltiplos) tipos de representações de objetos matemáticos;

- Exploração do caráter visual, dinâmico e manipulativo de objetos matemáticos;

- Incentivo a combinação de raciocínios intuitivo, indutivo e abdutivo, que podem contribuir ao desenvolvimento do raciocínio dedutivo;

- Criação de atividades matemáticas "abertas", ou seja, com direcionalidade ao seu objetivo;

- Ensinar e aprender Matemática de forma alternativa;

- Compreensão de conceitos; conhecimento de novas dinâmicas, formas de conectividade e relações de poder em sala de aula;

- Envolvimento com um novo tipo de linguagem (informática) na comunicação matemática, além da escrita;

- Criação de diferentes tipos símbolos e notações matemáticas;

- Aprofundamento em vários níveis de rigor matemático; identificação de incoerências conceituais e/ou aprimoramento de enunciado. (BORBA; SCUCUGLIA; GADANIDIS, 2014, p. 51-52).

Nesta pesquisa uma PMD foi produzida pelos participantes. Na realidade, por tratar-se de um videoclipe, foi produzida uma música (paródia - letra autoral) em uma das sessões do curso e, posteriormente, foi produzido um vídeo de conteúdo cênico/musical. Como mencionado, neste artigo optamos em discutir a produção dos GIFs que foram utilizados no videoclipe, ou seja, a produção das construções dinâmicas nas sessões iniciais do curso para posterior inclusão no roteiro da PMD por parte dos participantes. Diante de algumas características artístico-matemáticas apresentadas, os próprios GIFs podem ser também considerados PMDs.

A primeira Atividade do curso de extensão universitária propunha a construção de um triângulo equilátero (GIF 1) e de um tetraedro regular (GIF 2), ambos com características específicas. No caso do objeto bidimensional, sugerimos a construção de um segmento 
inscrito dinâmico paralelo a base. No caso do objeto tridimensional, propusemos a construção de um plano dinâmico paralelo a base. A Atividade 1 foi estruturada em 5 partes e está disponível em Anexo.

Resumidamente, após instruções para a construção dos objetivos, é proposta uma investigação acerca de diferentes valores para medidas de áreas de figuras que compõem a construção plana (triângulo + trapézio) e de volumes de sólidos que compõem a construção tridimensional (tetraedro + tronco de pirâmide). Notem que esses valores variam de acordo com a dinamicidade da construção por meio dos controles deslizantes. Por fim, é sugerida a criação dos GIFs e seu compartilhamento online em rede social. Na Figura 1 apresentamos exemplos dessas construções utilizadas na PMD dos participantes.
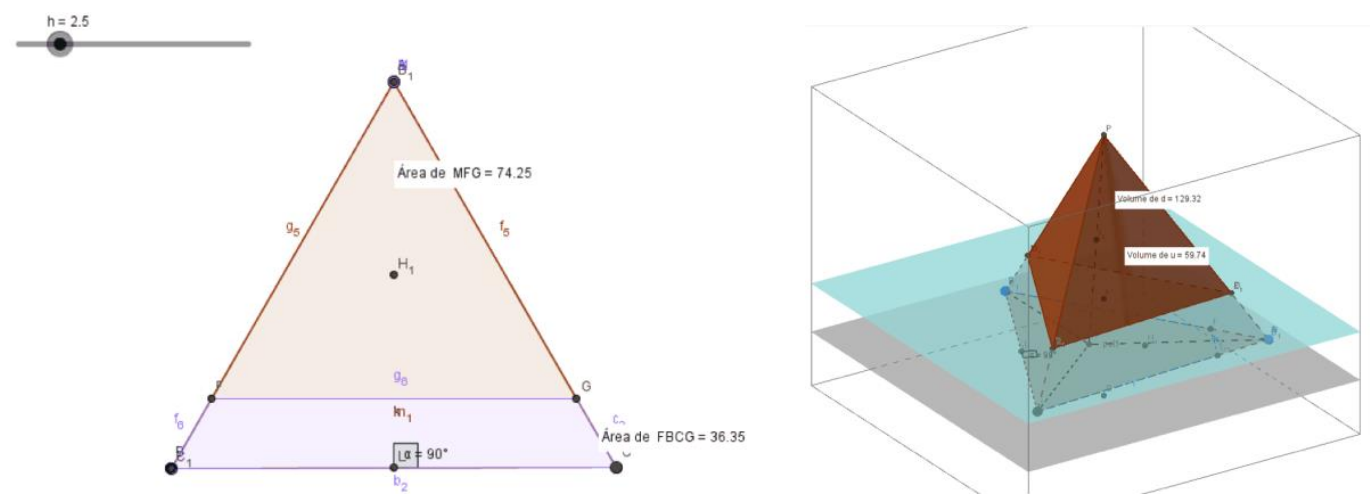

Figura 1: Exemplos de GIFs criados na pesquisa e utilizados no videoclipe (PMD). Fonte: Dados da pesquisa.

\section{Resultados e Discussão}

Para criar os GIFs, um aspecto importante foi o fato de os professores e futuros professores interagirem com o GeoGebra, possibilitando diversas formas de experimentação (VITAL, 2018). Os participantes também puderam explorar as construções, alterar as cores e o tamanho dos objetos, dentre outros elementos dinâmicos oferecidos pelo design do GeoGebra. Dessa maneira, o GeoGebra e os sujeitos da pesquisa se transformam, metaforicamente, em um coletivo de seres-humanos-com-GeoGebra, pois os atores tecnológicos e humanos realizaram uma moldagem recíproca (BORBA; VILLARREAL, 2005). Alguns aspectos artísticos também se destacaram, como a noção de movimento que os GIFs oferecem, pois

a repetição das imagens no cotidiano expande seu contato com os indivíduos, desta maneira, o leitor [de imagens] consegue ultrapassar a apresentação inicial na qual a imagem parece ser delimitada por seus elementos plásticos. Nesse movimento, o indivíduo passa a encontrar diferentes camadas de sentido (NADAL, 2014, p. 9).

Os elementos dinâmicos que compunham as construções no GeoGebra estavam interligados, ou seja, havia dependência entre os objetos da construção possibilitando ao usuário visualizar e estabelecer relações entre representações presentes na animação dos GIFs. De um ponto de vista artístico e matemático, uma característica importante dos GIFs construídos pelos participantes foi a simetria (ROHDE, 1977). A Matemática pôde, nesse 
sentido, ser explorada esteticamente. No caso dos GIFs 1 (triângulo) e GIFs 2 (tetraedro), foram identificados eixos e planos de simetria perpendiculares a base dos objetos. Além disso, os próprios GIFs se mostraram como PMDs, possibilitando que a Matemática fosse vista como algo atrativo e interativo, no sentido proposto por Gerofsky (2006, p. 2), de que é "incomum (e empolgante) vincular a Matemática e a Educação Matemática com performance, porque muitas das coisas que fazem as performances distintas e interessantes vão contra muitas das longas tradições da Matemática".

Particularmente, durante a primeira sessão do curso, o Grupo A explorou inicialmente o GIF 2, pois estavam com a janela de construção do tetraedro aberta quando chegaram na parte 5 da Atividade 1, a qual apresentava instruções para a construção dos GIFs. Mesmo as participantes do Grupo A tendo seguido as instruções na Atividade 1, o GIF 2 não funcionou adequadamente nessa situação. Quando o valor de $\mathrm{H}$ era alterado por meio do controle deslizante, nada dinâmico acontecia com a construção; nenhum segmento, ponto ou valor se modificava (Figura 2).
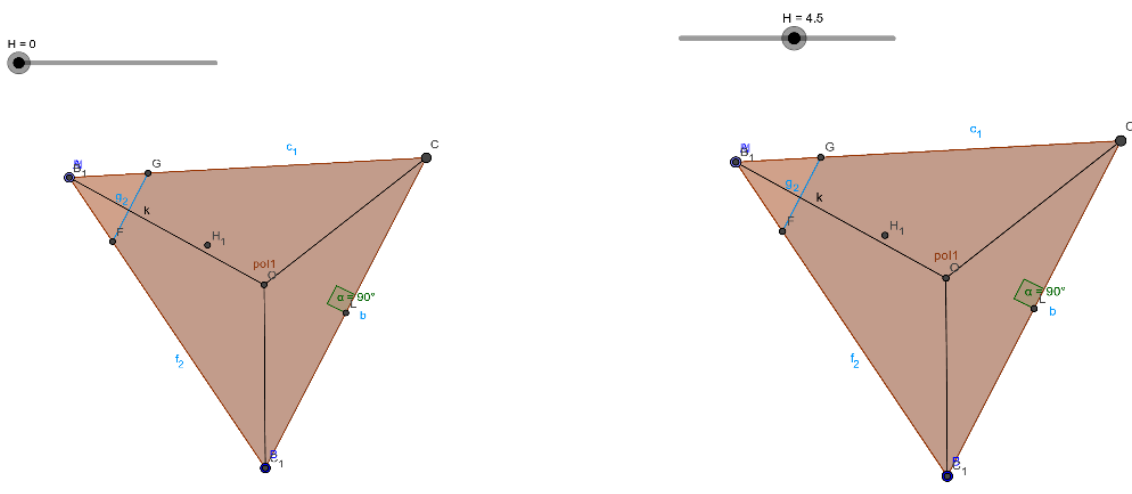

Figura 2: Construção do GIF 2A. Fonte: Dados da pesquisa.

No momento que as participantes do Grupo A abriram o arquivo GIF, perceberam que o objeto estava estático. No entanto, diante da sugestão proferida por Rita (mediadora), foi possível dar encaminhamento a uma solução diante do problema.

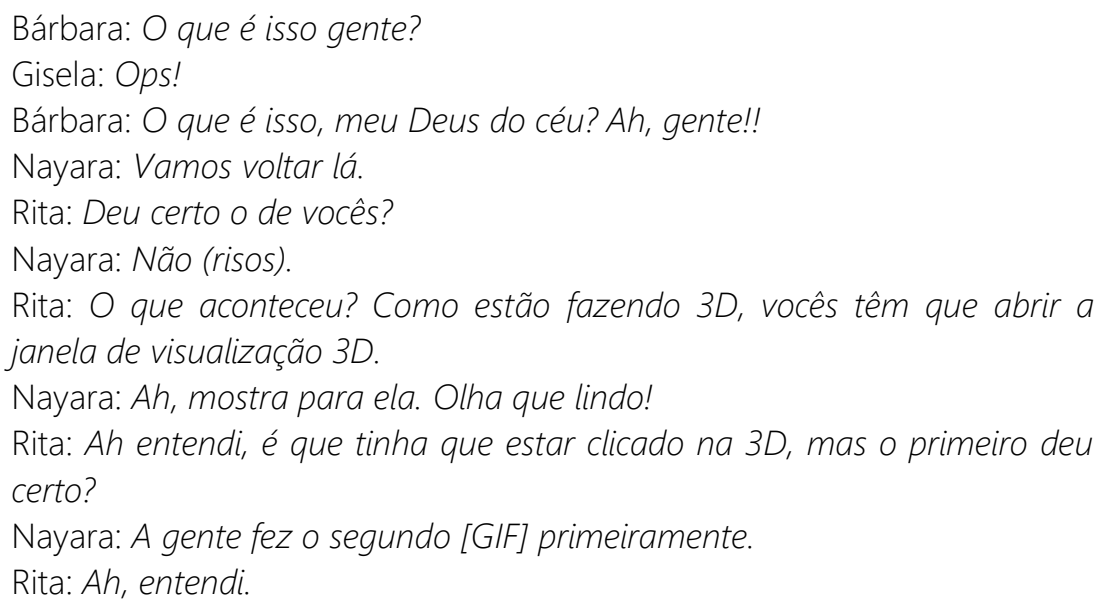

Em seguida, as participantes fizeram o que a Rita (mediadora) solicitou, abriram a janela de visualização 3D e criaram outro GIF, O GIF 2A. A reação delas foi de 
contentamento, considerando que anteriormente estavam "frustradas" por não terem realizado corretamente o GIF na primeira tentativa. O problema inicial na criação do GIF foi pelo fato de não terem aberto a janela de visualização 3D. Na realidade, o software gerou o GIF, porém apenas em duas dimensões, por isso o objeto não era disponibilizado tridimensionalmente. Ao abrirem a janela de visualização 3D, foi possível visualizar a construção tridimensionalmente.

O mesmo aconteceu com o Grupo C. Na construção realizada por esse grupo, o único elemento que variava era o valor de $\mathrm{H}$. O que se alterava na figura geométrica do GIF $2 \mathrm{C}$ era a cor do triângulo. Ao analisar o GIF criado por esse grupo, identificamos que os participantes não abriram a janela de visualização 3D, pois para fazer o GIF 3D é necessário que a janela de visualização 3D esteja aberta e selecionada. Portanto, selecionar corretamente a janela de visualização 3D no GeoGebra é um aspecto tecnológicopedagógico fundamental para criação de GIFs tridimensionais nesse software.

As integrantes do Grupo B testaram suas hipóteses com o intuito de descobrir algo por meio da experimentação com o GeoGebra, pois "a tecnologia tem um papel primordial em relação ao uso de experimentos em Matemática, bem como na Educação Matemática" (BORBA; VILLARREAL, 2005, p. 67). Os participantes puderam explorar construções e potencialidades diversas, testar várias hipóteses e elaborar conjecturas para produzir os GIFs. Assim, foi possível perceber que essa característica ficou evidenciada em todos os GIFs produzidos pelos sujeitos participantes da pesquisa. Com relação ao Grupo B, as integrantes também se mostraram engajadas na exploração da atividade, evidenciando em diversas situações aspectos heurísticos envolvendo o pensar-com-GeoGebra.

Laura: Você vem aqui nas propriedades do controle deslizante e ele é fixo ou aleatório.

Letícia: Ah, entendi.

Laura: Quando você o coloca no aleatório ele consegue, mas é só uma vez. Letícia: Ah, que legal, uma descoberta!

A utilização do controle deslizante foi uma experiência nova para os participantes, pois, apesar de alguns conhecerem o software, poucos conheciam esse recurso. Isto parece ter sido gratificante para eles, ou seja, criar algo que não haviam experimentado pedagogicamente ainda. Além disso, puderam testar hipóteses construídas ao longo do processo de criação dos GIFs, tanto a variação dos valores das áreas como dos volumes, indo ao encontro do que Borba e Villarreal (2005) defendem a respeito do processo de experimentação-com-tecnologia. Para eles "um experimento é realizado para descobrir algo desconhecido, para verificar a veracidade de uma hipótese, para aceitá-la ou rejeitá-la ou fornecer exemplos de uma verdade conhecida" (BORBA; VILLARREAL, 2005, p. 65).

Como mencionado, os GIFs foram produzidos nesta pesquisa para serem utilizados como elementos de uma narrativa digital na composição de uma PMD. Uma das características do modelo de PMDs conceituais (SCUCUGLIA, 2012) é a importância de trazer para o observador algo novo e surpreendente, pois isso possibilita a exploração de emoções e sensações à audiência, fomentando seu engajamento (visceral). "Os sentidos racionais e lógicos são importantes para a experiência de observação. Isto é, as surpresas devem ter sentido para serem efetivas" (SCUCUGLIA, 2012, p. 73). A seguir, apresentamos um diálogo do Grupo B sobre a "sensação de novidade", o que os levou a personalizar o GIF 2B de uma maneira diferente em relação às cores e à transparência dessas: 
Letícia: Vamos deixar bonito para enxergar direitinho.

Laura: Ah, coloca outra cor, que cor horrorosa!

Letícia: Tá estranho essa cor.

Laura: Ah, coloca outra vai, eu espero.

Letícia: Vamos mudar a cor da esfera, que tá muito ruim essa cor aqui dentro, não dá para ver, não tá aparecendo a esfera.

Laura: Deixa rosa.

As participantes alteraram a transparência e mudaram as cores do objeto de acordo com o que preferiram, para obter e oferecer melhor entendimento a respeito do que estavam construindo naquele momento. Notamos que estética e clareza na elaboração de um discurso matemático visual foram elementos convergentes. A Figura 3 mostra as cores que Letícia e Laura escolheram durante a construção do tetraedro regular no GeoGebra. Todos os elementos presentes estão em cores diferentes: os eixos do plano cartesiano, o tetraedro, a esfera, o plano sobreposto à base do tetraedro e o plano dinâmico.

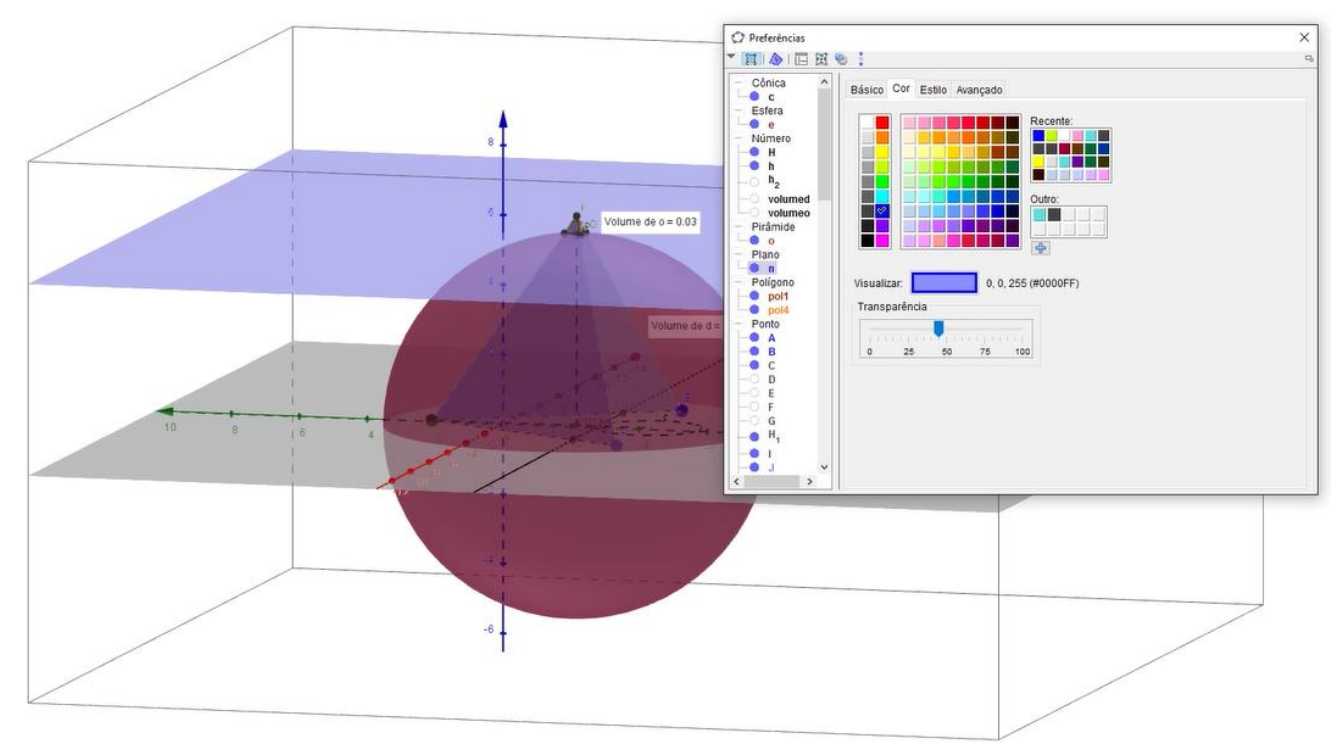

Figura 3: Alterações de cores para construção do GIF 2B. Fonte: Dados da pesquisa.

Os aspectos das cores são relevantes no sentido artístico, pois podem fomentar diferentes emoções e produção de sentidos (SCUCUGLIA, 2012), que remetem ao caráter multimodal do GIF, referente "à elaboração de significado que ocorre por meio da leitura, visualização, compreensão, resposta e produção e interação com textos multimídia e digitais" (WALSH, 2010, p. 213). Nos GIFs é possível identificar a representação visual geométrica das figuras e também os aspectos matemáticos referentes a cada uma delas, possibilitando que os sujeitos participantes da pesquisa utilizem "capacidades essenciais, como formular, empregar, interpretar e avaliar" (BRASIL, 2017, p. 275) na exploração de atividades matemáticas com tecnologias digitais.

Mesmo que as cores no GIF 2B estejam diferentes em relação à Figura 2, é importante destacar os processos de experimentação-com-tecnologias (BORBA; VILLARREAL, 2005) envolvidos na construção dos objetos. Cores foram modificadas por professores-comGeoGebra com o intuito de possibilitar a percepção/compreensão clara da construção por 
quem for visualizar e/ou utilizar o GIF criado. Dessa maneira, de um ponto de vista midiático e estético, há uma importante relação entre a tecnologia (o GeoGebra) e suas potencialidades (cores dos objetos) em termos de pensamento matemático.

O Grupo A foi o primeiro a concluir a primeira tentativa de construção do GIF 2, então optaram em modificar sua construção para que ela ficasse bidimensional e criar o GIF 1. Foram apagando ou ocultando elementos que consideraram "desnecessários" no GIF 1A em relação ao GIF 2A. Todo esse processo de testes possibilitou ao grupo fazer experimentações com o auxílio do GeoGebra. O resultado da construção é exibido na Figura 4. Triângulo e trapézio foram elaborados de forma que suas regiões e os valores de suas áreas ficassem visíveis.

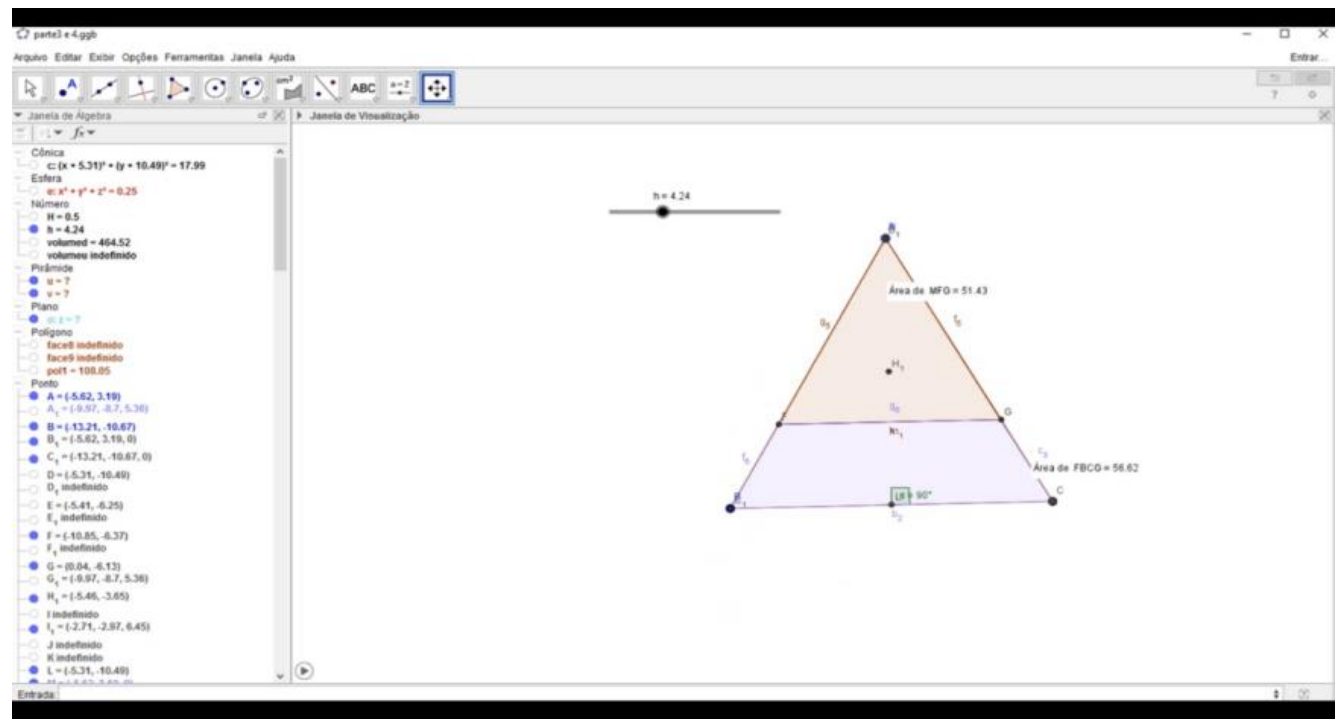

Figura 4: Construção do GIF 1A. Fonte: Dados da pesquisa.

Os outros dois grupos também fizeram o GIF 1. Um fator interessante foi a satisfação pedagógica do Grupo B nas falas de Letícia e Laura, destacadas a seguir, ao concluírem com sucesso a Atividade (Figura 5).

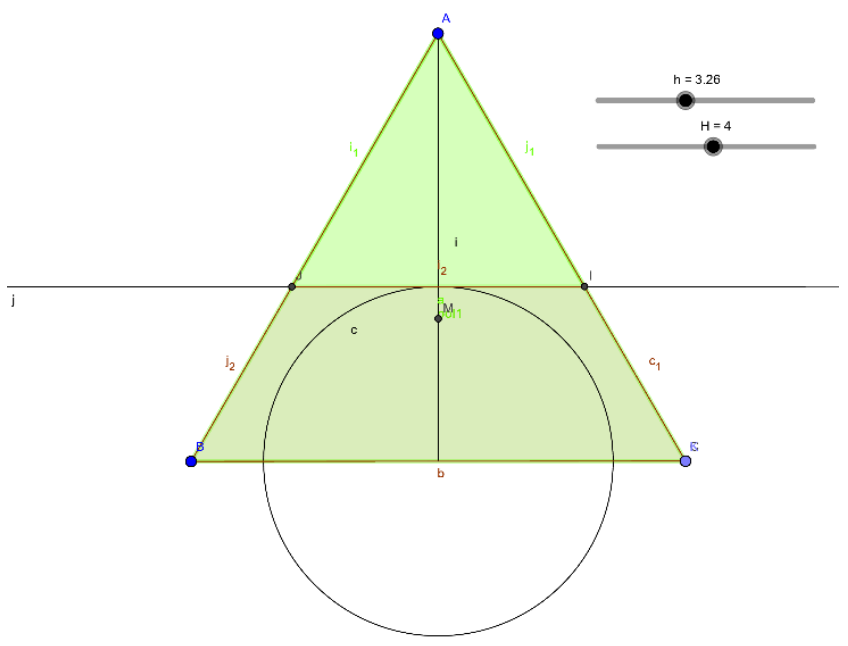

Figura 5: GIF 1 Grupo B. Fonte: Dados da pesquisa. 


\author{
Laura: Tomara que tenha dado certo. \\ Letícia: Ah, travou! \\ Laura: E agora? \\ Letícia: Eu vou fechar o GeoGebra e abrir de novo. Ah! Deu certo! \\ Laura: Agora foi, Letícia, o seu grito vai aparecer no áudio de todo \\ mundo.(...) Vamos postar no Facebook! \\ Letícia: No Facebook não fica muito bonito, né? Fica muito pequeno. Sabe \\ por que tem esse ponto aqui (ponto I)? \\ Letícia: Eu não sei por que tem esse ponto aqui. Lembra que eu falei que \\ não sabia para que servia esse ponto? Mas eu descobri, quer ver por quê? \\ Laura: Por quê? \\ Letícia: Porque nesse daqui [na construção 3D], tem um ponto bem no \\ meio, um ponto está ligado com o plano de baixo que toca o céu \\ [referindo-se à parte superior da construção].
}

O diálogo de Letícia e Laura é importante para identificarmos que foram diversas as maneiras como elas puderam experimentar e explorar algo desconhecido com o GeoGebra. Nos diálogos dos participantes da pesquisa é possível ver a interação que eles tiveram ao criar os GIFs. Segundo Borba e Villarreal (2005), ensinar de maneira tradicional é importante, mas também é desejável utilizar diferentes métodos para ensinar, como fazer perguntas ou discutir em grupos, como os sujeitos participantes da pesquisa fizeram. Eles dialogaram e testaram possibilidades com o auxílio do GeoGebra. No diálogo do Grupo B é possível perceber algumas características como "insights, conjecturas e crenças informais que estão conectadas à origem das descobertas matemáticas" (BORBA; VILLAREAL, 2005, p. 69, grifo dos autores). Nos GIFs apresentados, a visualização possui papel pedagógico importante, podendo ser compreendida de diferentes formas, desde a habilidade de interpretar informações e conseguir fazer uma representação mental, até construir a própria imagem num papel ou com uma noção espacial, por exemplo (BORBA; VILLARREAL, 2005).

\title{
Conclusões
}

Os GIFs 1 e GIFs 2 podem ser considerados elementos importantes da narrativa multimodal produzida pelos participantes na PMD, uma vez que oferecem informações visuais diversas relevantes à audiência. Eles têm potencial para oferecer emoções vicárias (BOORSTIN, 1990), trazendo sensações para a audiência, principalmente pela combinação de cores e pelos movimentos rápidos e dinamicidade presentes na animação (WEINER, 2010). A característica visceral é peculiar aos GIFs, sendo complexa as descrições acerca dos tipos de sensações/emoções que o público poderá sentir ao iniciar a reprodução de GIFs de qualquer natureza. Há uma evidente dimensão intersubjetiva no trabalho com GIFs.

Além disso, a ideia Matemática/Geométrica explorada na PMD por meio dos GIFs pode ser considerada relevante do ponto de vista conceitual, visto que os principais aspectos presentes nos GIFs são as relações exploradas na Atividade 1: GIF 1 - relações numéricas/visuais envolvendo as áreas do "triângulo menor" e do trapézio que formavam o triângulo equilátero; GIF 2 - a relação entre os volumes dos sólidos gerados pelo plano dinâmico que "dividia" o tetraedro em um "tetraedro menor" e um tronco de pirâmide. Esse aspecto matemático é importante para a compreensão e solução do problema, pois os GIFs podem facilitar a compreensão e resolução da Atividade 1 por meio da visualização devido 
à síntese de informações contidas nele. Os GIFs apresentam informações compiladas de maneira simples, mesmo o processo de construção sendo complexo. Artisticamente, é possível identificar aspectos das formas geométricas e das cores que são significativos para a compreensão matemática. Além disso, é destacada a sensação de movimento de looping realizado pelos GIFs, pois essa mídia permite comunicar ideias de maneira direta e rápida, atribuindo complexidade ao processo de visualização (WEINER, 2010; NADAL, 2014).

A natureza multimodal da comunicação nesse cenário criativo (BORBA; SCUCUGLIA; GADANIDIS, 2014; WALSH, 2010) une-se aos aspectos artísticos dos GIFs, pois são comunicadas ideias por meio de diferentes modos como, por exemplo, o movimento, a noção de espacialidade das construções geométricas, a visualização e a representação numérica e geométrica. Nesse caso, o que se destaca na atividade como um todo é a presença e relação entre formas geométricas (dinâmicas) e do valor de suas áreas e volumes. A surpresa matemática emergiu para os participantes da pesquisa no cenário de investigação principalmente por meio da utilização do controle deslizante, o qual fez com que o segmento nos GIFs1 e o plano nos GIFs2 se movimentem, alterando o valor das suas áreas e volumes e permitindo a criação dos GIFs. Também, o compartilhamento de GIFs em ambientes virtuais, incluindo redes sociais, atribui significância pedagógica-social à produção matemática de estudantes e professores. Nesse caso, produtos educacionais que compilam aspectos da inteligência matemática-artística coletiva de professores e futuros professores foram compartilhados em loci público virtual para além dos confins da sala de aula de um curso de extensão universitária. As produções matemáticas-digitais dos participantes do curso estão disponíveis em ambientes online para estudantes, professores e outros interessados nessas temáticas (Geometria, GeoGebra, GIFs, etc.).

Compreendemos que a partir da construção e interação dos sujeitos com os GIFs, ou seja, por meio do processo de pensar-com-GIFs, os coletivos de seres-humanos-comGeoGebra identificaram detalhes nos problemas propostos (variação de cores, "arrasto dinâmico", ocultação de elementos dos objetos, etc.). A possibilidade de animação/movimentação do GIF pôde moldar a maneira com a qual os professores e futuros professores de Matemática perceberam o problema, pois conforme o valor da medida da área do triângulo e do trapézio variavam, a posição do segmento de reta que estava no "triângulo maior" também variava. Analogamente, no GIF 2, é importante destacar a relação entre o volume do tetraedro regular e do outro sólido em relação ao plano que os dividia. À medida que o plano se deslocava para cima, os volumes do tetraedro regular e do tronco, respectivamente, diminuíam e aumentavam; da mesma maneira, quando o plano se deslocava para baixo acontecia o inverso com os seus volumes. Esses fatores unem-se a possiblidade de experimentação que tal construto oferece, visto que as tecnologias condicionam a produção de conhecimentos (matemáticos). Neste caso, o GeoGebra e suas potencialidades proporcionaram a construção dos GIFs, constituindo um coletivo pensante de seres-humanos-com-GeoGebra.

\section{Agradecimentos}

CNPq - Conselho Nacional de Desenvolvimento Científico e Tecnológico (428323/2018-9). CAPES - Coordenadoria de Aperfeiçoamento de Pessoal de Nível Superior. 


\section{Referências}

ALVES; C. M. Animação de Movimentos e Repetição: Uma ênfase nas possibilidades do formato GIF - Universidade Estadual Paulista, São Paulo, 2015.

ARAGÃO, R. M. R.; GONÇALVES, T. O. Vamos introduzir práticas de investigação narrativa no ensino de matemática?!. Amazônia: Revista de Educação em Ciências e Matemáticas, v. 1, p. 121-128, jun. 2005.

BICUDO, M. A. V. Pesquisa Qualitativa: significados e a razão que a sustenta. Revista Pesquisa Qualitativa, São Paulo, v. 1, n. 1, p. 7-26. 2005.

BOORSTIN, J. The Hollywood Eye. What makes movies work. New York: Cornelia \& Michael Bessie Books, 1990.

BORBA, M.; SCUCUGLIA, R.; GADANIDIS, G. Fases das tecnologias digitais em Educação Matemática: sala de aula e Internet em movimento. Belo Horizonte: Editora Autêntica, 2014.

BORBA, M. C.; VILLARREAL, M. E. Humans-With-Media and the reorganization of Mathematical Thinking: information and communication technologies, modeling, experimentation and visualization. New York: Springer, 2005.

BRASIL. Secretaria de Educação Básica. Base Nacional Comum Curricular: Educação é a Base. Brasília; Ministério da Educação. 2017.

DOXIADIS, A. Embedding mathematics in the soul: narrative as a force in mathematics education, Opening address to the Third Mediterranean Conference of Mathematics Education, Athens, Greece, 2003.

FARIA, R. W. S. de C.; ROMANELLO, L. A.; DOMINGUES, N. S. Fases das tecnologias digitais na exploração matemática em sala de aula: das calculadoras gráficas aos celulares inteligentes. Amazônia: Revista de Educação em Ciências e Matemáticas, [S.I.], v. 14, n. 30, p. 105-122, out. 2018.

GADANIDIS, G.; BORBA, M. C. Digital Mathematical Performance. http://www.edu.uwo.ca/dmp, 2006.

GEROFSKY, S. Performance Time \& Space. In: DIGITAL MATHEMATICS PERFORMANCE SYMPOSIUM, 2006, Ontario. Anais... London, ON: UWO, 2006. p. 1-11.

GREGORUTTI, G. S. Performance Matemática Digital e imagem pública da Matemática: viagem poética na formação inicial de professores. 2016, 163 f. Dissertação (Mestrado em Educação Matemática) - Universidade Estadual Paulista, Júlio de Mesquita Filho, Rio Claro, 2016.

LACERDA, H. D. G. Educação Matemática encena. 2015, 179 f. Dissertação (Mestrado em Educação Matemática). Universidade Estadual Paulista, "Júlio de Mesquita Filho", Rio Claro, 2015.

MORAES, J. C. P. de. A teoria da forma de Kandinsky: uma compreensão artística e subjetiva de elementos geométricos. Amazônia: Revista de Educação em Ciências e Matemáticas, [S.I.], v. 16, n. 35, p. $45-60$, abr. 2020. 
NADAL, J. H. D. A Cultura do GIF: reconfigurações de imagens técnicas a partir dos usos e apropriações de narrativas cíclicas. 2014. 174 f. Dissertação (Mestrado em Comunicação e Linguagens) - Universidade Tuiti do Paraná, Curitiba, 2014.

POWELL, A. B.; FRANCISCO, J.; MAHER, C. Uma abordagem à análise de dados de vídeo para investigar o desenvolvimento das ideias Matemáticas e do raciocínio de estudantes. BOLEMA, Boletim de Educação Matemática. Rio Claro, v.17, n. 21, p.81-140, maio. 2004.

ROHDE; G. M. Simetria: Rigor e imaginação. Porto Alegre: EDIPUCRS, 1977.

SCUCUGLIA, R. On the nature of students' digital mathematical performances: When elementary school students produce mathematical multimodal artistic narratives. Alemanha: Verlag/Lap Lambert Academic Publishing, 2012.

SCUCUGLIA, R.; GADANIDIS, G. Performance Matemática: Tecnologias Digitais e Artes da Escola Pública de Ensino Fundamental. In: BORBA, M. C.; CHIARI, A. S. S. (Org.). Tecnologias Digitais e Educação Matemática. 1. ed. São Paulo: Livraria da Física, p. 325-363. 2013.

SCUCUGLIA, R. Narrativas multimodais: a imagem dos matemáticos em performances Matemáticas digitais. BOLEMA, Boletim de Educação Matemática. Rio Claro, v. 28, n. 49, p. 950-973, ago. 2014.

SCUCUGLIA, R. R. S.; GREGORUTTI, G. S. Explorando o Teorema das Quatro Cores em Performances Matemáticas Digitais. BoEM, Boletim online de Educação Matemática, Joinville, v.3. n.5, p. 2-17, ago./dez. 2015.

SCUCUGLIA, R. R. S. The pedagogic role of the arts and digital media in the practice of the Onario Mathematics Curriculum. BOLEMA, Boletim de Educação Matemática. Rio Claro, v. 29, n. 53, p 1043-1065, dez. 2015.

SINCLAIR, N. The roles of the aesthetic in mathematical inquiry. Mathematical Thinking and Learning, v. 6, n. 3, p. 261-284, nov. 2004.

STAKE, R. E. Investigación con estudio de casos. Madrid: Morata, 1999.

URDANETA, S. C. D; GONZÁLEZ, J. P.; CASTILLO, A. D. V. D. Interpretação geométrica dos signos das razões trigonométricas com Geogebra. Amazônia: Revista de Educação em Ciências e Matemáticas, [S.I.], v. 13, n. 28, p. 78-89, dez. 2017.

VITAL, C. Performance Matemática Digital e GeoGebra: possibilidade artístico-tecnológica em Educação Matemática. 2018, 136f. Dissertação (Mestrado em Educação Matemática) Universidade Estadual Paulista, "Júlio de Mesquita Filho". Rio Claro, 2018.

WALSH, M. Multimodal literacy: What does it mean for classroom practice? Australian Journal of Language and Literacy, v. 33, n. 3, p. 211-223, 2010.

WEINER, J. Christina Hendricks on an Endless Loop: The glorious GIF renaissance, 2010. Disponível em: http://www.slate.com/articles/arts/culturebox/2010/10/christina_ hendricks_on_an_endless_loop.html. Acesso em 18 abr. 2017.

ZALESKI FILHO, D. Matemática e Arte. 1. ed. Belo Horizonte: Autêntica, 2013. 